Supplementary information

\title{
Rational Design of Highly Potent, Selective and Bioavailable SGK1 Protein Kinase Inhibitors for the Treatment of Osteoarthritis
}

Nis Halland, * Friedemann Schmidt, ${ }^{*}$ Tilo Weiss, Ziyu Li, Jörg Czech, Joachim Saas, Danping Ding-Pfennigdorff, Matthias K. Dreyer, Carsten Strübing and Marc Nazare*† Integrated Drug Discovery, Sanofi R\&D, Industriepark Höchst, D-65926 Frankfurt am Main, Germany. †Leibniz-Institut für Molekulare Pharmakologie (FMP), RobertRössle-Straße 10, 13125 Berlin-Buch, Germany.

Contact: Nis.Halland@Sanofi.com

\section{Table of contents}

NMR and LCMS data $\quad$ S2

$\begin{array}{ll}\text { Kinase panel } & \mathrm{S} 11\end{array}$

Protein crystal structure determination $\quad \mathrm{S} 12$

Thermodynamic solubility determination $\quad \mathrm{S13}$ 


\section{NMR and LCMS data}

N-[4-(3-Amino-1H-pyrazolo[3,4-d]pyrimidin-6-yl)-phenyl]-5-chloro-2,4-difluorobenzenesulfonamide $\mathbf{9 a}$

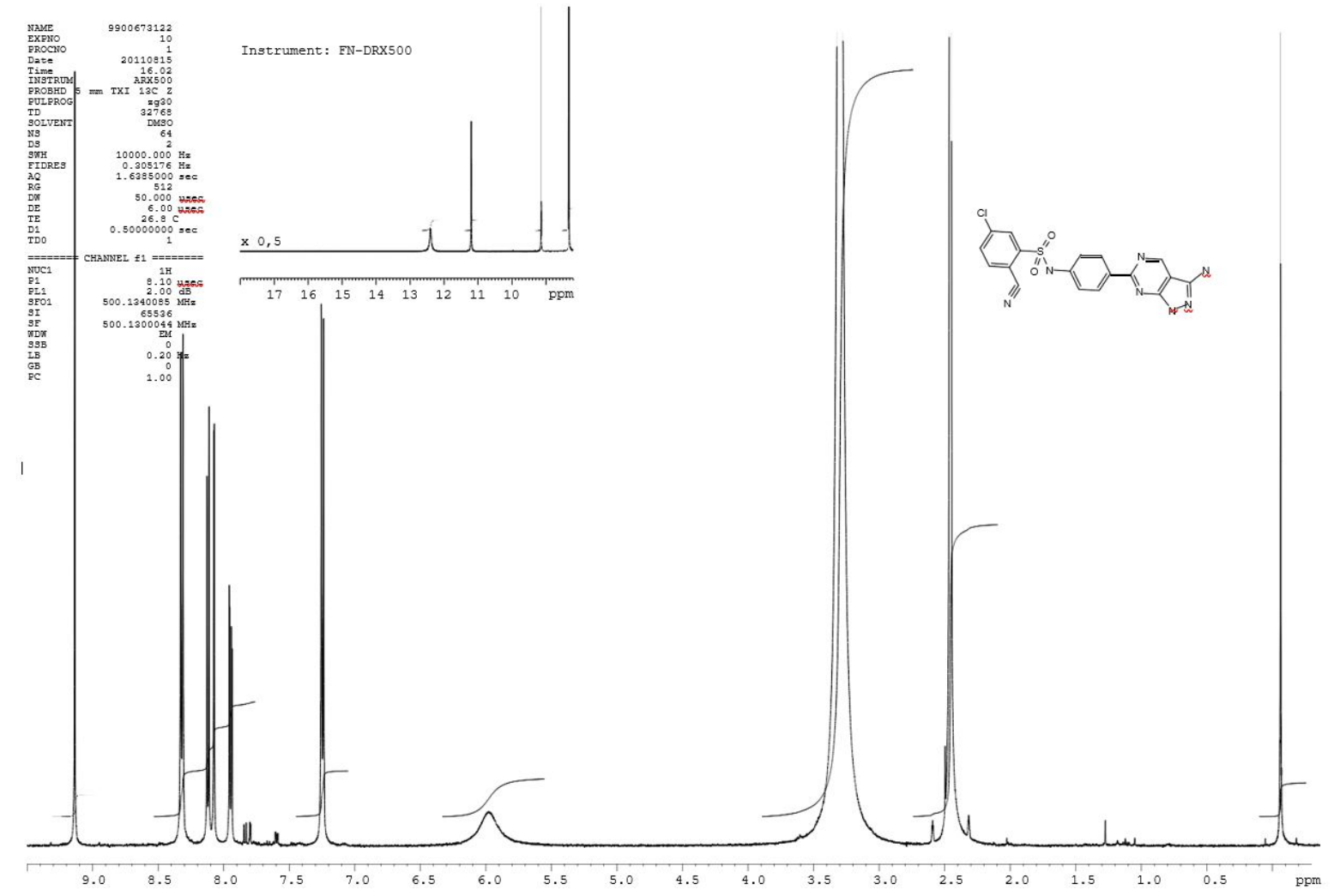


Openlynx Report Openlynx Report Sanofi-Aventis Frankfurt, Discovery Analytics Mass spectrometry G878 LAB_NAZ Page 1

Sample: 1

File:9900673122

Description:FFC.EQY6.229

A: $\mathrm{H} 2 \mathrm{O}+0.05 \% \mathrm{FA} \mathrm{B}: \mathrm{ACN}+0.035 \% \mathrm{FA}$

Vial:1:5,C

Date:15-Aug-2011

Submitter:LAB_NAZARE

$0.9 \mathrm{ml} / \mathrm{min}$
ID:

Time:15:59:30

UPLC SQD:ACQ-SQD\#C09SQD448W

Grad: $98: 2$ (0min) to 5:95 (2min) 5:95 (2.6min)

Printed: Mon Aug 15 16:04:38 2011

Sample Report:

3: UV Detector: TIC

$1.987 e+2$

$\mid \begin{gathered}16) \\ 100 \%\end{gathered}$

425.0 (98\%)

425.0 (95\%)

1.61

虽

$1.5 e+2$
$1.0 e+2$
$5.0 e+1$
0.0

Peak Number
6

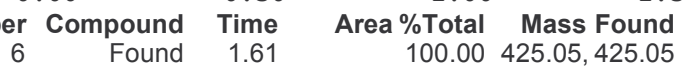

Range: $1.987 \mathrm{e}+2$

3: UV Detector: 220

1.314

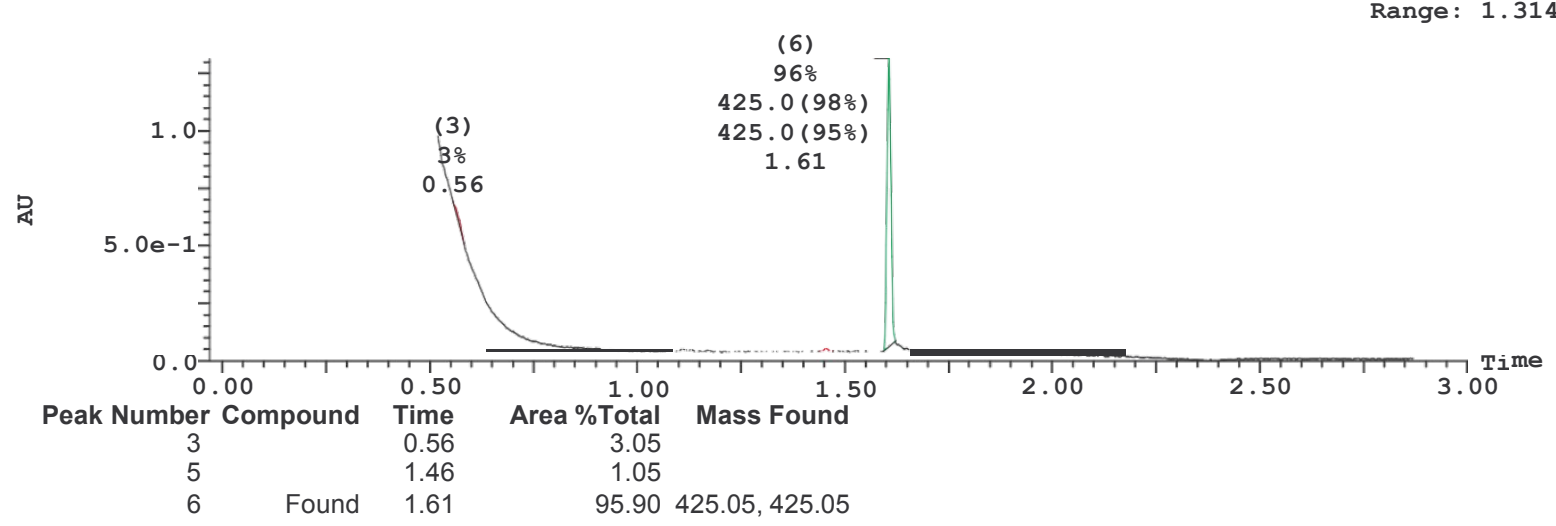

3: UV Detector: 254

1.687

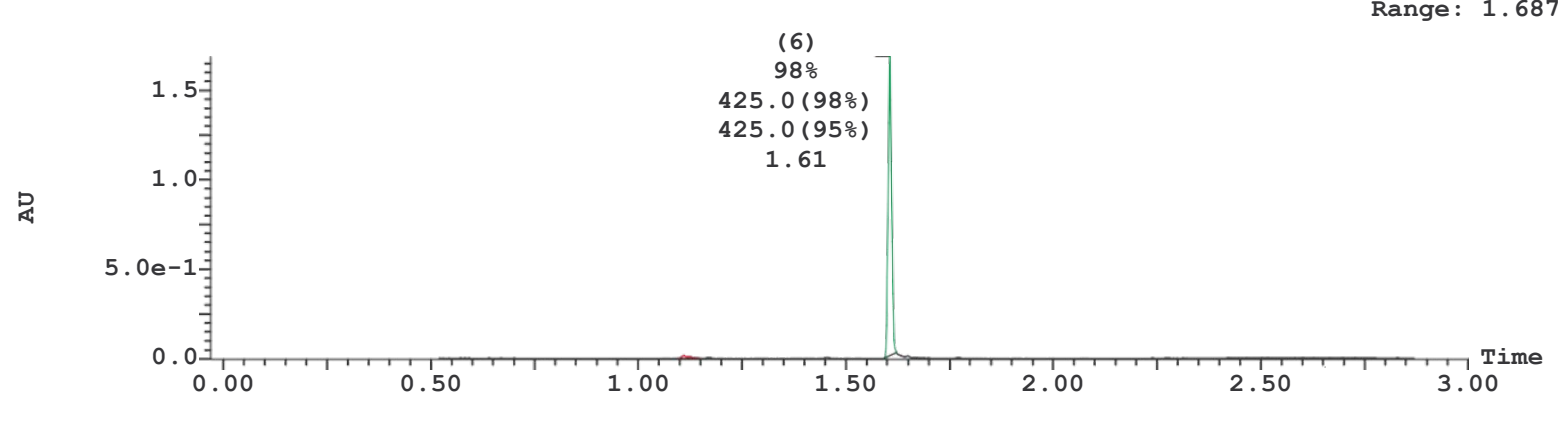


Sample Report (continued):

\section{Peak ID Compound Time Mass Found}

$6 \quad$ Found 1.60 Mass Found

6:(Time: 1.61) Combine (335:339-(303:313+361:370)) 1:MS ES+

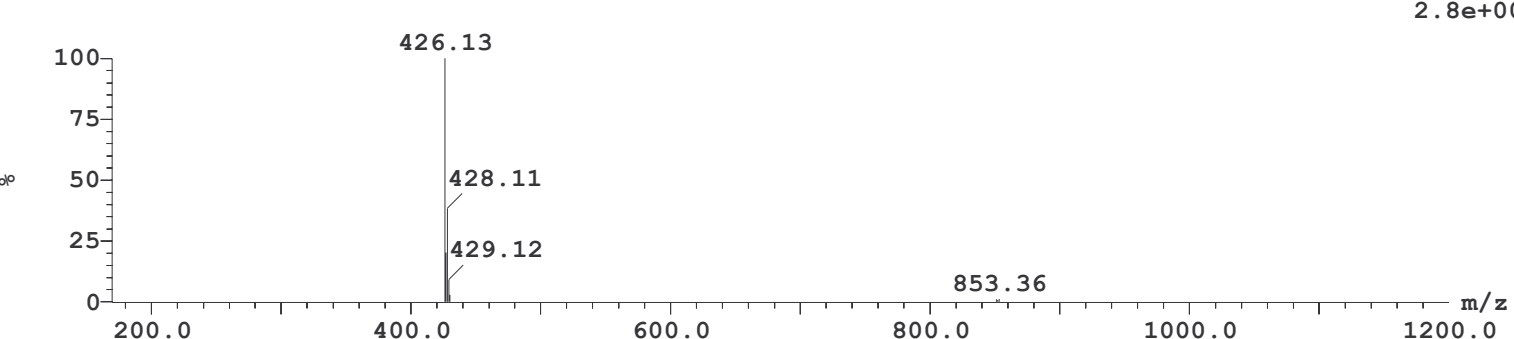

Peak ID Compound Time Mass Found

6: (Time: 1.61$)$ Combine $(334: 338-(303: 312+360: 370)$ )

$2:$ MS ES-

424.19

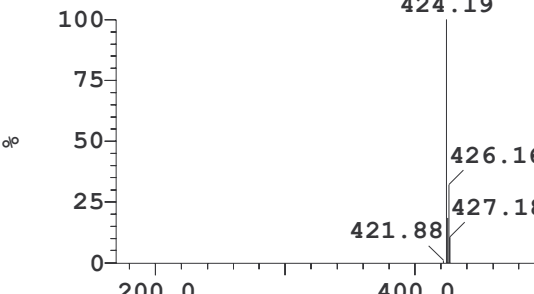

574.23

853.00

$4.9 \mathrm{e}+005$

200.0

400.0

600.0

800.0

1000.0

1200.0 
5-chloro-2-fluoro-N-[4-[4-[(1-isopropyl-4-piperidyl)oxy]-3-methyl-1H-pyrazolo[3,4d]pyrimidin-6-yl]phenyl]benzenesulfonamide $\cdot \mathrm{HCl}$ 16y ( 1:1 conformation mixture).

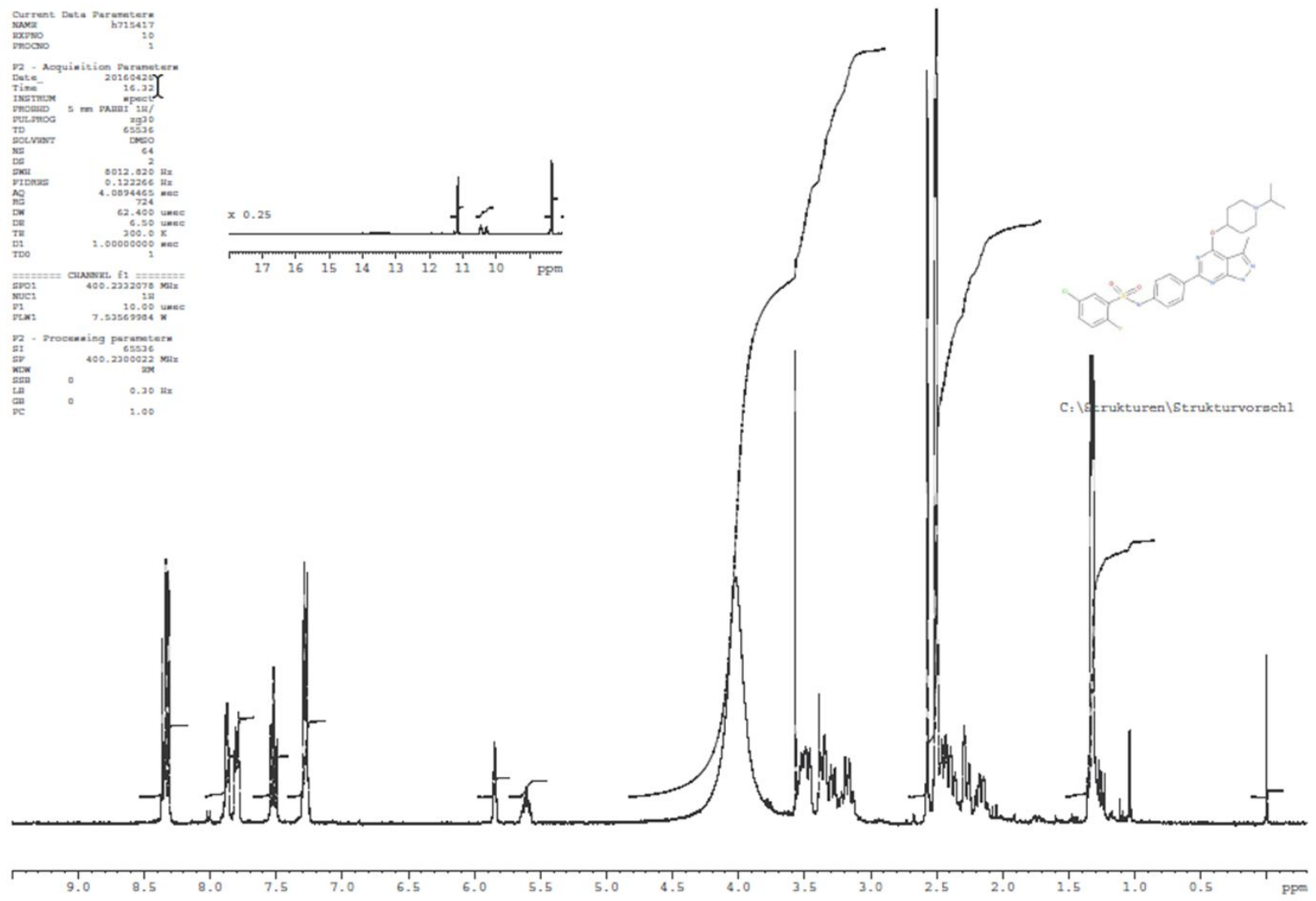


Sample Report:

3: UV Detector: TIC

$7.657 e+1$

(3)

$98 \%$

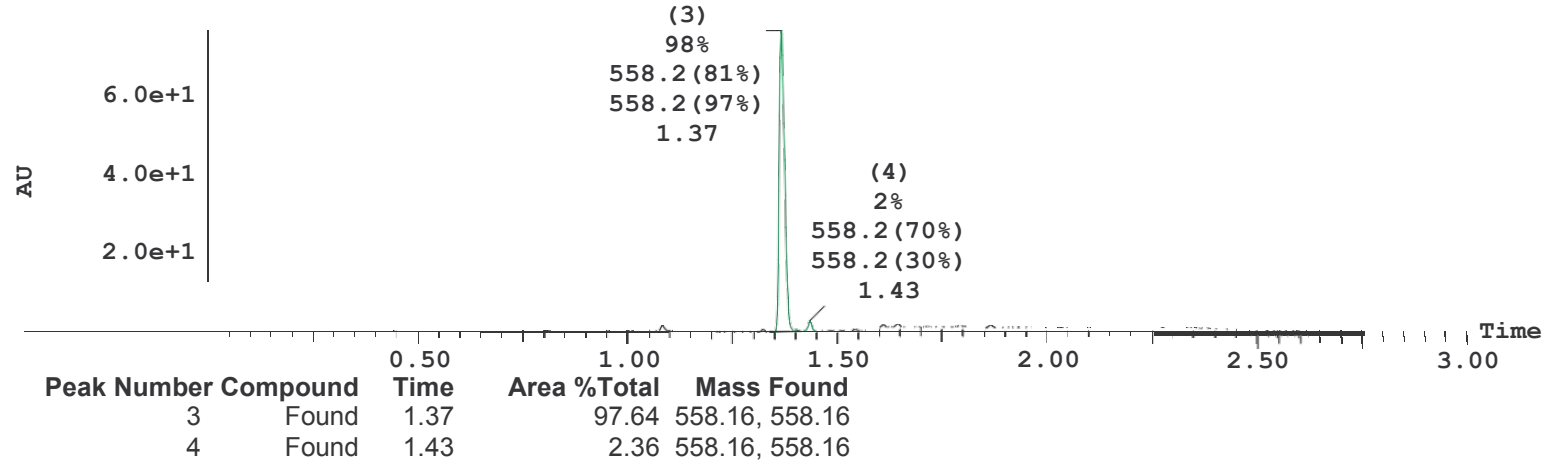

3: UV Detector: 220

$8.733 e-1$

Range: $8.706 \mathrm{e}-1$

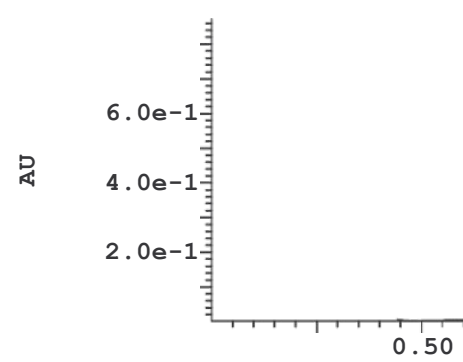

Peak Number Compound Time

$\begin{array}{lll}2 & & 1.08 \\ 3 & \text { Found } & 1.37 \\ 4 & \text { Found } & 1.43 \\ 5 & \text { Found } & 1.64\end{array}$

(3)

$95 \%$

$558.2(81 \%)$

$558.2(97 \%)$

1.37

(4)

$3 \%$

$558.2(70 \%)$

$558.2(30 \%)$

1.43

3: UV Detector: 254

$5.427 e-1$

Range: $5.426 \mathrm{e}-1$

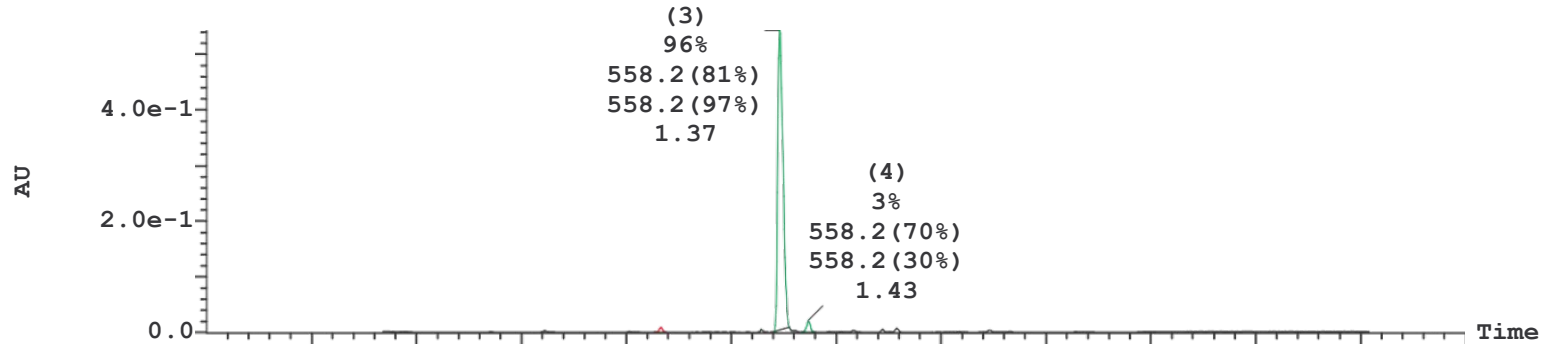


Sample Report (continued):

Peak ID Compound Time Mass Found

3 Found $1.38 \quad 559.16$

3: (Time: 1.39) Combine $(269: 273-(240: 249+294: 302)) \quad 1: M S$ ES+

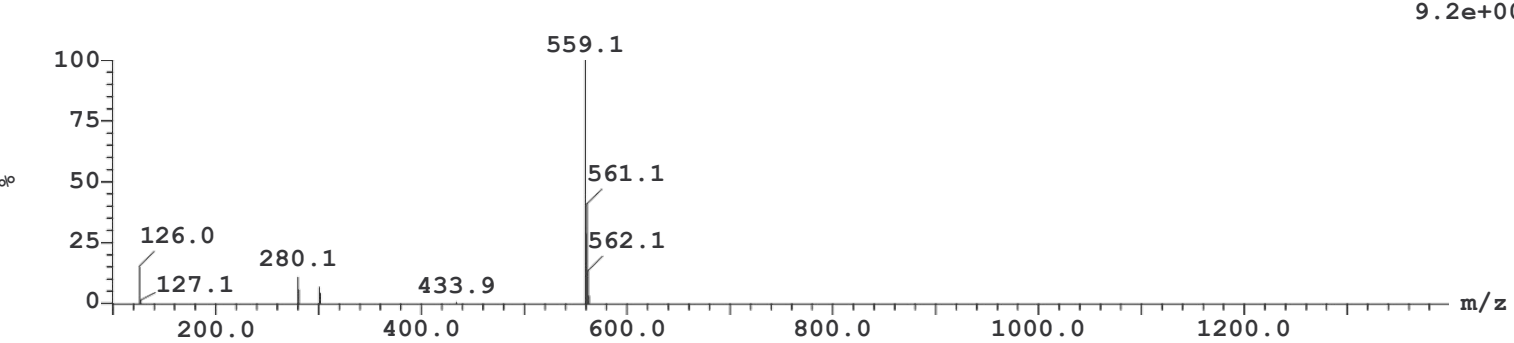

$\begin{array}{rrrr}\text { Peak ID } & \text { Compound } & \text { Time } & \text { Mass Found } \\ 3 & \text { Found } & 1.38 & 557.16\end{array}$

3: (Time: 1.39) Combine (269:273-(239:248+293:302)) 2:MS ES-

(559.1


5-chloro-2-fluoro-N-(4-(4-((4-hydroxycyclohexyl)oxy)-1H-pyrazolo[3,4-d]pyrimidin-6yl)phenyl)benzenesulfonamide hydrochloride 17

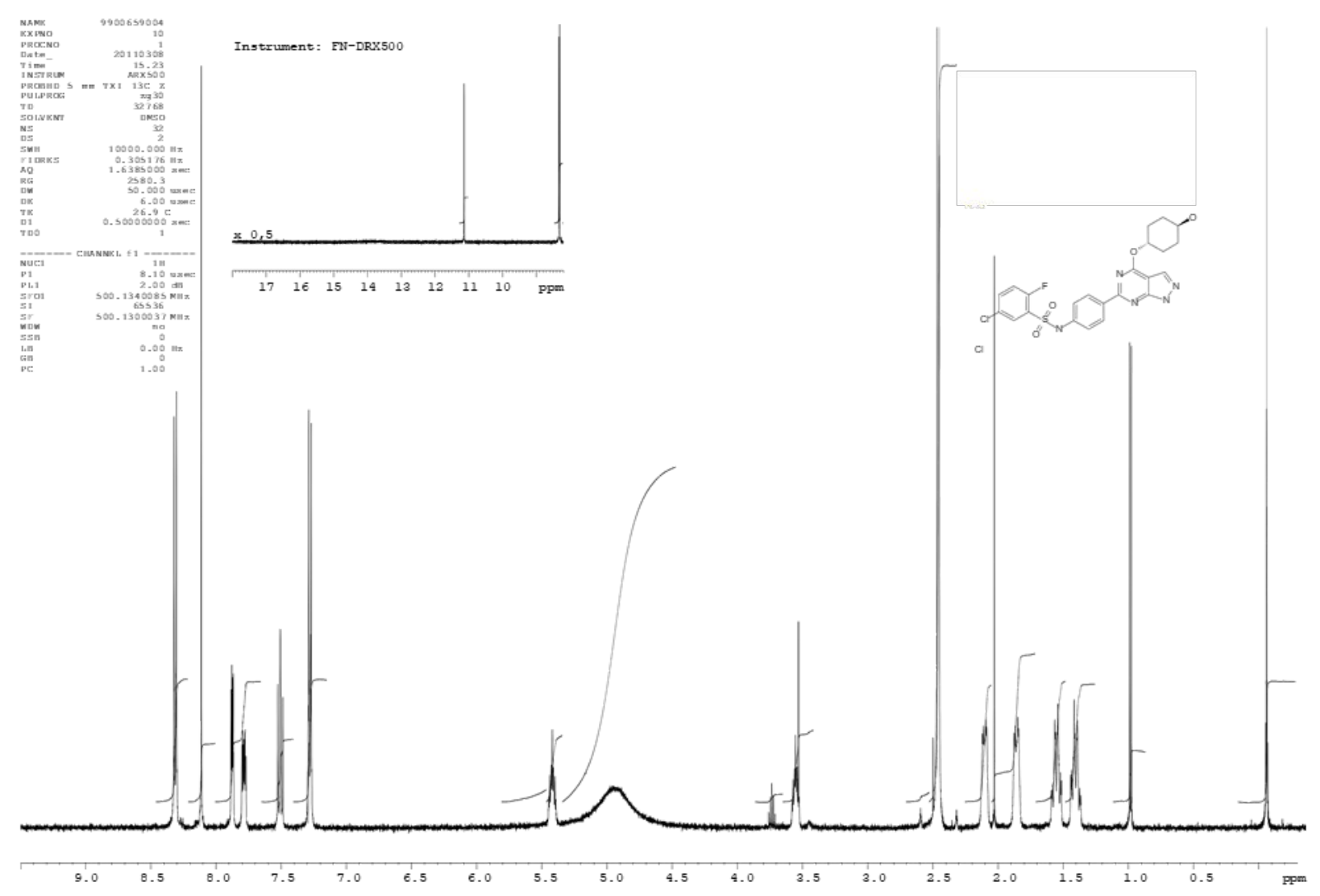


Sample Report:

3: UV Detector: TIC

2. $685 e+2$

Range: $2.663 e+2$

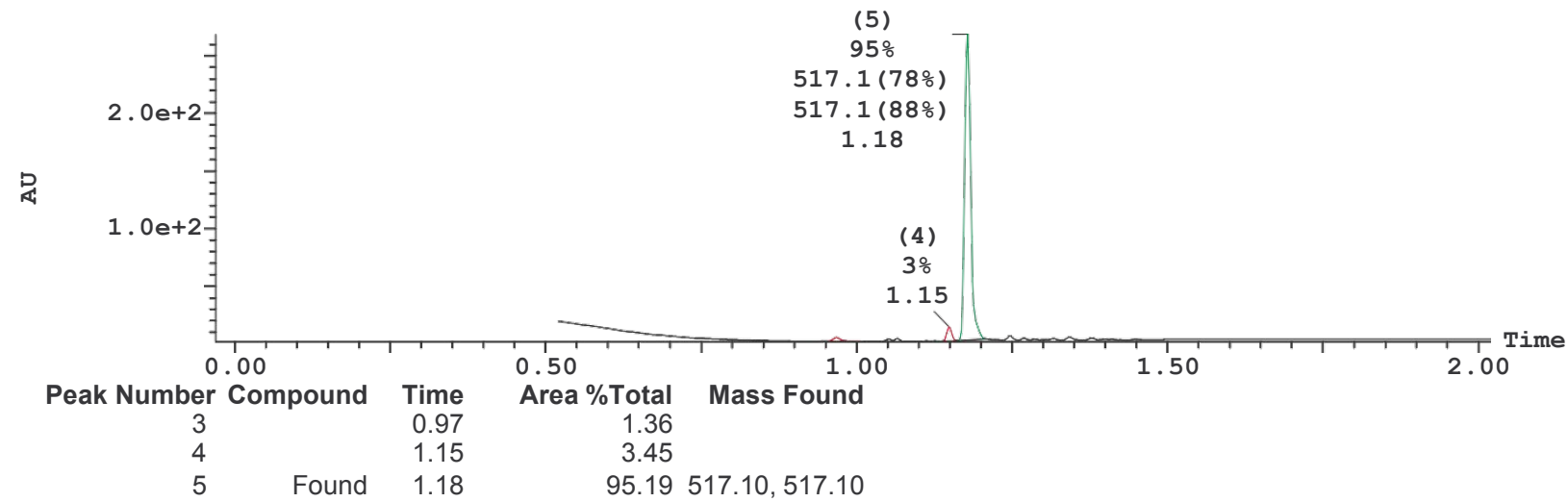

3: UV Detector: 220

1.988

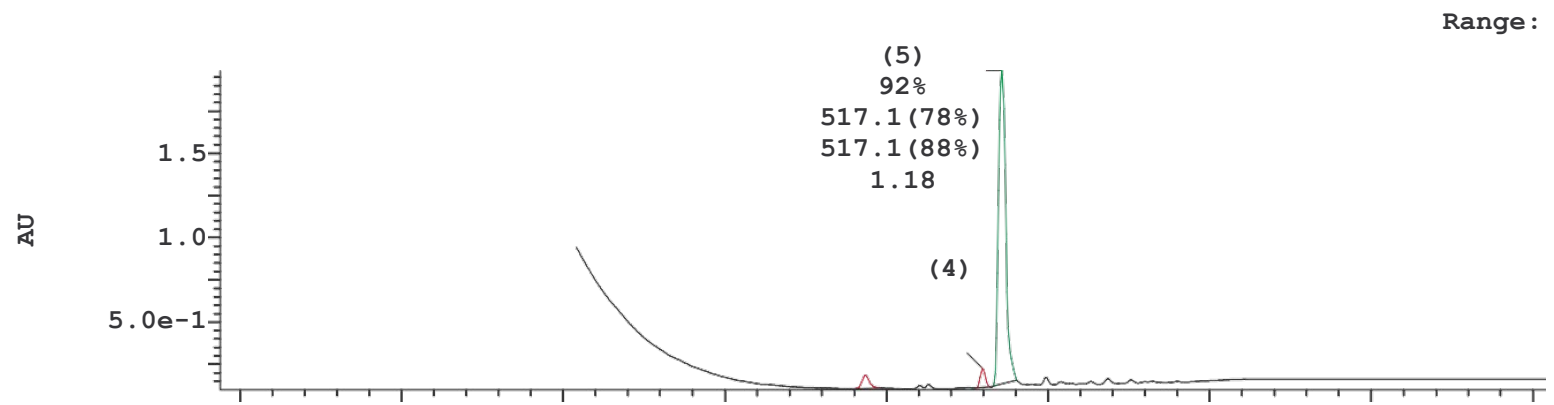

Sample Report (continued):

3: UV Detector: 254

(5)

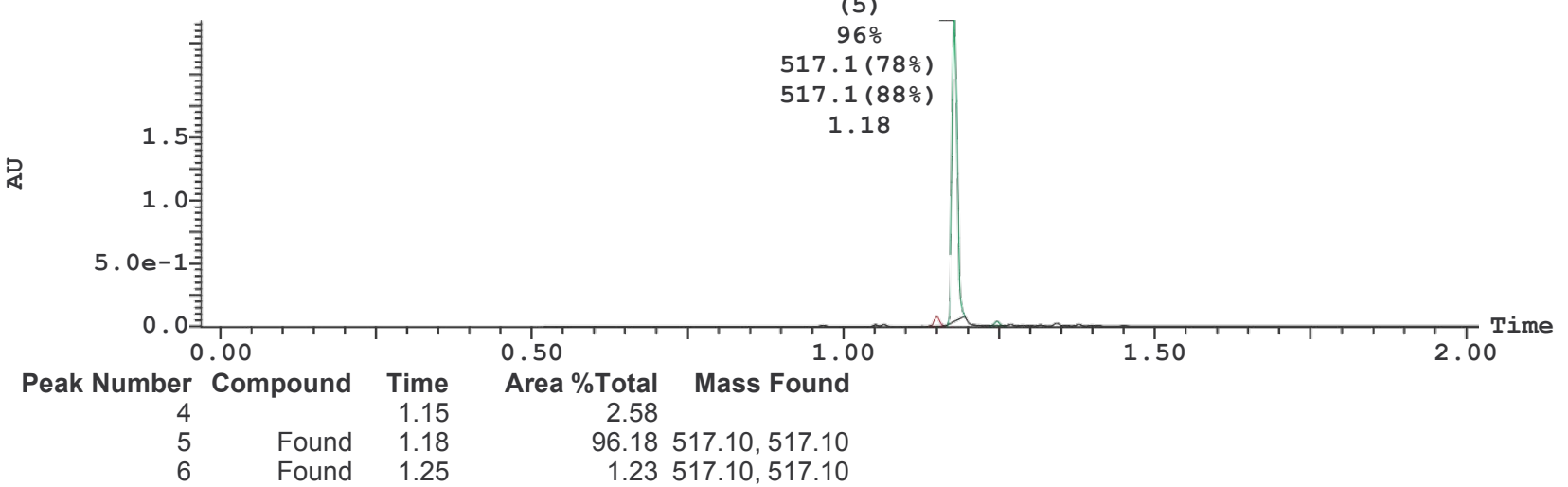


Peak ID Compound Time Mass Found

$5 \quad$ Found $1.17 \quad 518.10$

5: (Time: 1.17) Combine (243:247-(212:221+269:278))

$1:$ MS ES+

4. $7 e+006$

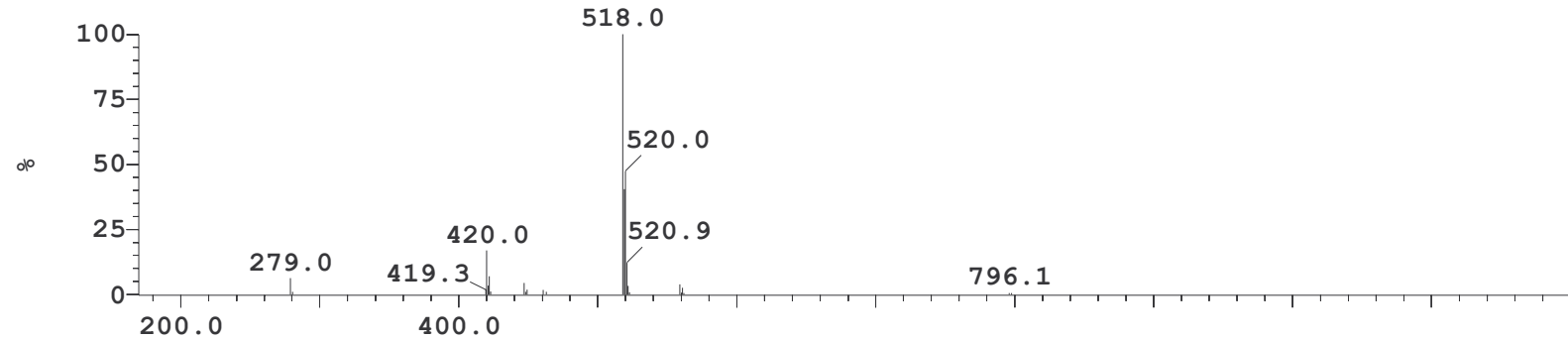

Peak ID Compound Time Mass Found

\section{$5 \quad$ Found $1.17 \quad 516.10$}

5: (Time: 1.17) Combine $(243: 247-(212: 221+269: 278)$

2 :MS ES-

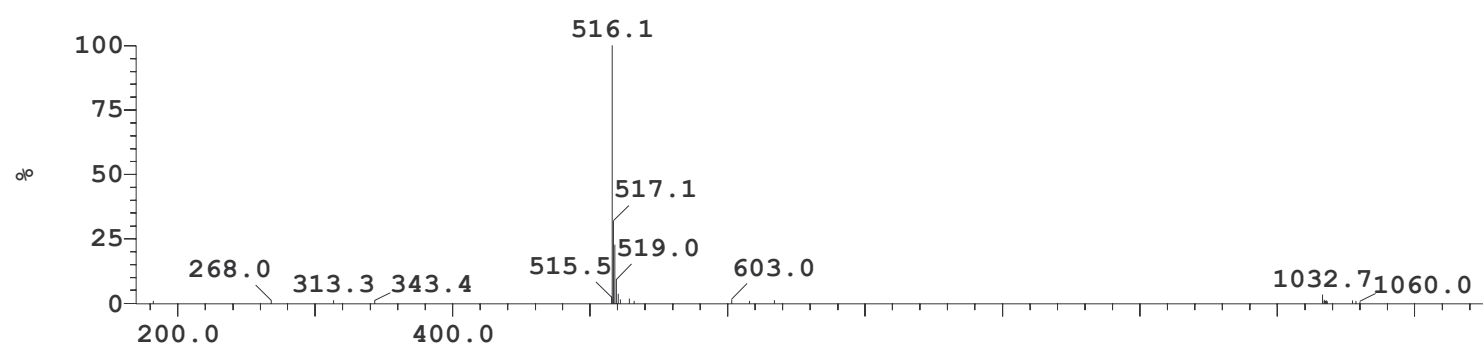




\section{Kinase panel}

Compound were tested against the following 60 kinases at $0.1 \mu \mathrm{M}$ concentration and 2Km ATP concentration.

Table S1. List of kinases in test panel

\begin{tabular}{|c|c|c|c|c|}
\hline$\overline{A B L}$ & INSR & PIM2 & LCK & CamK1a \\
\hline AKT1 & JAK3 & cRAF & LYN & LRRK2(G2019S) \\
\hline $\begin{array}{l}\text { VEGFR2- } \\
\text { KDR }\end{array}$ & $\begin{array}{l}\text { AMPK- } \\
\text { alpha2/beta1/gamma1 }\end{array}$ & EPHA1 & MET & PEK(EIF2AK3) \\
\hline AurB & BRSK2 & EPHA3 & $\begin{array}{l}\text { MST1R } \\
\text { (RON) }\end{array}$ & CDK5 \\
\hline KIT & CDK9 & EPHB1 & P70S6K & LRRK2(G2019S) \\
\hline $\begin{array}{l}\text { CAMK2 } \\
\text { delta }\end{array}$ & MST2 & EPHB4 & PAK4 & DDR2 \\
\hline MELK & CDC2/Cycline B1 & Erk1 & PIM1 & HcK \\
\hline p38a & DYRK1B & FLT1 & ALK & $\mathrm{PKCb} 2$ \\
\hline CLK2 & PDGFR_alpha & Flt3 & BTK & DDR1 \\
\hline $\begin{array}{l}\text { p38-gamma } \\
\text { (MAPK12) }\end{array}$ & PKA & GSK3b & RSK1 & PKCepsilon \\
\hline EGFR & PKCz & $\begin{array}{l}\text { GSK3- } \\
\text { alpha }\end{array}$ & $\begin{array}{l}\text { IKBKE } \\
\text { (IKK } \\
\text { epsilon) }\end{array}$ & TRKA(NTRK1) \\
\hline FGFR1 & PLK1 & SRC & SYK & PKC Theta \\
\hline
\end{tabular}




\section{Protein crystal structure determination}

Table S2: Crystallographic data collection and refinement statistics*

*values in parentheses refer to the highest resolution shell

\begin{tabular}{|l|l|}
\hline Space group & P6 22 \\
\hline Cell dimensions a,c $(\AA)$ & $92.4,172.0$ \\
\hline Resolution $(\AA)$ (highest resol. shell) & $19.54-2.51(2.77-2.51)$ \\
\hline$R_{\text {merge }}$ & $0.074(1.388)$ \\
\hline$R_{\text {meas }}$ & $0.079(1.497)$ \\
\hline$R_{\text {pim }}$ & $0.038(0.541)$ \\
\hline Total no. of observations & $92642(3711)$ \\
\hline Total no. unique & $10903(546)$ \\
\hline Mean I/s(l) & $19.3(1.5)$ \\
\hline Completeness (\%) & $92.9(69.3)$ \\
\hline Multiplicity & $8.5(6.8)$ \\
\hline CC $1 / 2$ & $1.000(0.713)$ \\
\hline$R_{\text {work }} / R_{\text {free }}$ & $0.2015 / 0.2443$ \\
\hline Protein atoms (non-H) & 2166 \\
\hline R.m.s deviations bond lengths, angles & $0.009 \AA, 1.13^{\circ}$ \\
\hline Average B-factor protein atoms $\left(\AA^{2}\right)$ & 87.2 \\
\hline Average B-factor atoms ligand $3 a\left(\AA^{2}\right)$ & 78.7 \\
\hline
\end{tabular}




\section{Figure S1}

Difference electron density omit map calculated after deletion of ligand $3 a$ from the final refined structure. The map, contoured at $3.0 \sigma$ and superimposed on the refined ligand, clearly shows the position and orientation of the inhibitor.

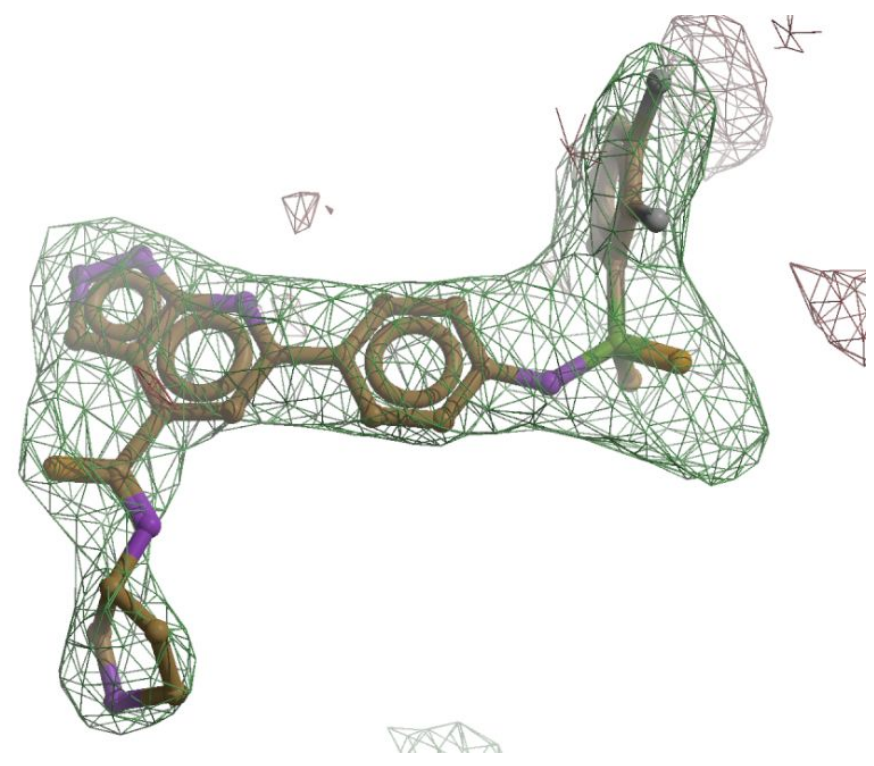

Thermodynamic solubility determination based on 10mM DMSO stock solution

Instrument: $\quad$ Waters Acquity UPLC with Binary Pump

Software

Waters Empower 3 (Build 3471)

Column:

Waters Acquity CSH C18 $1.7 \mu \mathrm{m} 2.1$ x $75 \mathrm{~mm}$

Eluent A:

Water $+25 \mathrm{mM}$ Ammonium Acetate

Eluent B:

Acetonitrile

\section{Gradient:}

\begin{tabular}{|c|c|c|c|c|}
\hline Time [min] & Flow Rate $[\mathbf{m L} / \mathbf{m i n}]$ & $\mathbf{\% A}$ & $\mathbf{\% B}$ & Slope \\
\hline 0 & 0.9 & 98 & 2 & \\
\hline 0.05 & 0.9 & 98 & 2 & linear \\
\hline 2.30 & 0.9 & 2 & 98 & linear \\
\hline 2.95 & 0.9 & 2 & 98 & linear \\
\hline 3.0 & 0.9 & 98 & 2 & linear \\
\hline
\end{tabular}


$\begin{array}{ll}\text { Detection: } & \text { UV at } 220 \mathrm{~nm} \\ \text { Column } & 50{ }^{\circ} \mathrm{C} \\ \text { temperature: } & \end{array}$

DMSO stock solutions were plated in a 96 well plate with $100 \mu \mathrm{g}$ compound in each well and DMSO was evaporated. $160 \mu \mathrm{L}$ buffer and a magnetic bead was added to each well and the plate sealed and shaken for 16 hours at $25^{\circ} \mathrm{C}$ (Eppendorf thermomixer with $600 \mathrm{rpm}$ ). The plate was centrifugated for $5 \mathrm{~min}$ at $400 \mathrm{o}$ rcf and filtered through $0.45 \mu \mathrm{m}$ low-binding hydrophilic PTFE (Millipore). The supernatant was analysed UPLC-UV $220 \mathrm{~nm}$ and the concentration determined based on peak area-to-real concentration ratio from previous calibration run.

\section{Composition of aqueous buffer media}

\begin{tabular}{|c|l|}
\hline Medium & \multicolumn{1}{|c|}{ Composition } \\
\hline Phosphate buffer pH 7.4 & $\begin{array}{l}40.5 \mathrm{~mL} \mathrm{Na}_{2} \mathrm{HPO}_{4} 0.1 \mathrm{M}+9.5 \mathrm{~mL} \mathrm{NaH} \mathrm{PO}_{4} 0.1 \mathrm{M} \\
\text { solution adjusted to } 100.0 \mathrm{~mL} \text { with Millipore water }\end{array}$ \\
\hline FeSSIF pH 5.0 & Biorelevant Media FeSSIF pH5.0 \\
\hline FaSSIF pH 6.5 & Biorelevant Media FaSSIF pH6.5 \\
\hline
\end{tabular}

